\title{
Feed Forward Control of Welding Process Parameters Through On-Line Ultrasonic Thickness Measurement
}

\author{
Momchil Vasilev $^{1 *}$, Charles MacLeod ${ }^{1}$, Yashar Javadi ${ }^{1}$, Gareth Pierce ${ }^{1}$, Anthony Gachagan ${ }^{1}$ \\ ${ }^{1}$ Centre for Ultrasonic Engineering (CUE), Department of Electronic \& Electrical Engineering, \\ University of Strathclyde, Glasgow G1 1XQ, UK
}

*Corresponding author's email: momchil.vasilev@strath.ac.uk

\begin{abstract}
Accurate control of welding parameters, such as arc power and wire speed, is critical for successful and appropriate weld joint penetration. The thickness of components being welded is a key parameter in defining optimum arc power for successful penetration, but is not always known beforehand or well controlled in manual and automated welding operations. This paper presents a novel feed-forward, sensor enabled, arc process control strategy based on ultrasonic thickness measurement. An ultrasonic wheel probe, deployed simultaneously alongside the welding torch, enables plate thickness measurement when coupled with a low-latency thickness measurement algorithm deployed on a real-time controller. An automated GTAW fusion control strategy, based on wheel probe measured plate thickness, provides direct input control of arc current, welding torch travel speed and filler wire feed rate. A parametric relationship between ultrasonically measured sample thickness and key arc welding parameters for welding S275 mild steel samples of thickness between $4.1 \mathrm{~mm}$ and $6.1 \mathrm{~mm}$ was established and verified. It is demonstrated that the system can measure and derive plate thickness while adapting arc power in real-time, with sufficiently lowlatency, to allow consistent weld seam and uniform penetration on variable thickness steel samples, which under normal open-loop circumstances lead to sample burn-through and excessive penetration.
\end{abstract}

\section{Introduction}

It is imperative to accurately control the power and energy of fusion welding operations to ensure optimum weld penetration, integrity and process efficiency. Incorrect or non-optimum welding parameters lead to potential undesirable effects such as distortion, defects, Heat-Affected Zone (HAZ) abnormalities, wasted energy and overall production inefficiency. In traditional fusion welding and manufacturing operations, welders optimize arc power, torch travel speed and wire feed rate manually dependent on parameters such as welding process, material, part thickness, and welding torch orientation [1]. This technique forms one part of the occupations overall high skill requirement and subsequently the profession faces a predicted $60 \%$ gap worldwide in suitable worker availability in the next decade [2].

Fusion welding has been widely adopted in many high-value manufacture industries, including aerospace, automotive, defence and energy. Fusion welding is a process for permanently joining 
pieces of material of similar or dissimilar nature in a variety of configurations ranging from simple butt to butt connections to more complex shapes, e.g. pipes, petrochemical vessels and ship hulls. In simpler manufacturing operations, knowledge of constant part thickness allows pre-setting of input parameters and arc power, based on pre-loaded part thickness/welding power synergic curves or operator experience. When considering complex manufacturing, operations with parts of varying thickness requires either accurate pre-measurement, control and fixturing, or operator skill in manual operations to compensate for such variations. Examples of applications involving changing and differing part thickness include design-optimised structures, where light-weighting through the use of thinner sheets is desired alongside high-strength thicker sections in key areas - These would include multi-thickness sheet arrays used in modern large-section fabrications in the shipbuilding, energy and defence sectors. Additional applications, in both manufacturing and asset repair applications, include tapered sections and varying thickness joints due to manufacturing and plate tolerances, corrosion and thickness loss.

While the manufacturing sector and welding productivity in the UK and worldwide has, on the whole, increased significantly year-on-year since the 1970s, manufacturing growth has remained generally stagnant throughout, leaving labour numbers to be reduced significantly to remain competitive [3]. Therefore, for future manufacturing and welding to increase productivity, remain globally competitive and to achieve growth to meet demand in key areas related to transport [4], [5] and energy [6], new approaches in manufacturing concepts and solutions are crucial. New developments in "Smart Manufacturing" technological areas, such as sensors, robotics, automation and control, play a fundamental critical role in offering solutions to these grand challenges and greatly advancing the High Value Manufacturing (HVM) of advanced welded components.

Considering the current industrial revolution (4.0) [7] and forward, the future HVM process must embrace a wholly interdisciplinary approach, encompassing core disciplines such as manufacturing, mechanical, electronic and control engineering, enabling sensor driven automation of traditional manufacturing operations to improve their accuracy, repeatability and speed. The current industrial state-of-the-art automated fusion welding approaches largely consist of either column and boom gantries or 6 Degree of Freedom (DOF) robotic manipulators equipped with controllable welding power sources. Fusion Welding processes such as Gas Metal Arc Welding (GMAW), Gas Tungsten Arc Welding (GTAW) and Submerged Arc Welding (SAW) process are commonly utilised for tasks such as plate, pipe and seam welding, cladding and additive structure building [8].

Automated fusion welding systems are often plagued with issues such as gap width variation inflexibility, component and electrode tip misalignment and wire feed problems [8] and hence require additional sensors to mitigate these adverse factors. When considering automated applications, modern fusion power sources offer a number of control and programming options, of both a digital and analogue nature dependent on the application and of basic parameters such as arc current (GTAW), arc voltage (GMAW), wire speed (GMAW and automated GTAW) and various other more advanced parameters. Knowledge of the thickness of both parts to be welded is of significant importance when considering optimum welding parameters and subsequent arc power in order to avoid weld flaws and to ensure a high production efficiency [9]. This can be pre-programmed or inputted to some modern automated welding systems alongside careful part, process and placement tolerance and control. However, such approaches do not support flexible manufacturing or repair operations where tolerances, unconstrained part placement or part or process tolerances are unknown or varying. Furthermore, the welding torch travel speed would also need to adapt to the changes in material thickness and hence welding current, to ensure adequate arc energy is 
maintained throughout. Finally, the wire feed rate would also need to accommodate changes in part thickness and travel speed in order to supply a consistent flow of filler material to the joint.

Typical optical sensor integrations for on-line weld monitoring and can be split into passive, when consisting of only optical sensors, and active, when a structured light source, usually a laser or a projector, is used in conjunction with the optical sensors. Simpler solutions combine a single camera with image processing, e.g. edge detection, to accurately measure the width of the weld pool [10]. In [11] authors demonstrate a more advanced approach that utilizes an infrared camera and an artificial neural network to measure the weld bead width and depth based on the measured surface temperature of the workpiece. With regards to active optical systems, a projected laser line can be used to both track the weld seam [12] and to measure the profile geometry of the produced weld bead for detection of defects such as weld bead misalignment, undercut and plate displacement [13]. In [14] such a laser line is projected behind the weld pool in a GTAW butt-weld configuration and the arc current and arc length are closed-loop controlled in order to maintain a complete weld penetration. Due to the distance between the weld pool and the laser line, however, certain delays were present in the control loop. Alternatively to a laser line, a laser dot pattern can be projected on the weld pool. The dot pattern that is reflected off the weld pool surface can be acquired using a camera and used to accurately reconstruct and measure the 3D surface of the weld pool [15]. It has been demonstrated that by measuring the weld pool length, width and convexity of the weld pool surface, the weld penetration can be estimated in real-time [16] and the weld pool surface can be controlled in a closed-loop [17]. While optical laser systems have been shown to detect and compensate for a variety of weld disturbances such as variations in gas flow rate, root opening and heat transfer conditions, they have not been deployed on samples with thickness variations. Furthermore, as all optical methods require a direct line of sight to the weld pool and weld seam, they are not suitable for some applications like SAW.

Automated thickness measurement of the parts to be joined during the welding operation presents the opportunity to ensure adaptive and optimum welding parameters for the parts under fusion. Such a technique offers benefits for both manual and automated welding operations in terms of final product integrity, production rate and reduced re-work. The opportunity exists to employ a thickness measurement system to provide feed-forward control of the welding parameters in samples of changing thickness. Such as system would require a lower computational complexity when compared to structured light systems that employ 3D calculations and machine learning. A feed-forward thickness measurement could be integrated with a feed-back control system, using suitable weld sensors such as structured light systems [17] or ultrasonic phased array [18] so that the deposited weld can be measured, verified and controlled, providing optimum process automation and part quality. Furthermore, an on-line thickness measurement system would complement a weld penetration control system as an additional sensory unit, as the thickness measurements would provide an accurate setpoint for the desired penetration depth.

Although optical laser systems can provide joint preparation profile information and subsequently infer part thickness in open joint configurations, when considering close knit or butt joint configurations, such surface inspection techniques are often unable to provide reliable part thickness information. Furthermore, such weld bead and weld pool measurement techniques provide useful sensory inspection information on the surface, but they do not perform any internal or sub-surface measurement. In separate industrial applications such as post-build Non-Destructive Evaluation (NDE), internal imaging of parts and components is commonly undertaken using ultrasonic sensor techniques and not only provide internal information on the part but also, with knowledge of the speed of sound in the material, the thickness of the part. Such wall thickness 
measurements can be taken in-situ and can be utilized for process control [19], [20] in a feedforward architecture for optimised weld penetration for a given plate thickness.

Thickness mapping is used extensively in inspection and NDE applications where measured information relates to corrosion loss and remaining life of the components and structures. The two main methods for thickness mapping are the ultrasonic and Eddy Current (EC) techniques [21], [22]. $\mathrm{EC}$, and more recently Pulsed EC, methods based on the electromagnetic induction principle ascertain sample thickness through a variation of measurement coil inductance. EC testing is fully non-contacting (attractive for welding process), but sensitive to changes in lift-off distance and local changes of both material conductivity and permeability [23], [24]. Furthermore, the high electric current and EMI required for fusion welding (usually in the range $100 \mathrm{~A}$ to $300 \mathrm{~A}$ ) is likely to interfere with the low-current EC measurements. Traditional Ultrasonic Thickness (UT) sensors on the other hand, are particularly suitable for thicker materials. Through reference calibration against a known thickness, and the fact that probe frequency and material wave velocity is nominally constant at a given temperature, the thickness of the material can be ascertained from back-wall time-of-flight measurement. Such thickness measurement instruments are commercially available with high accuracy options reaching a resolution of $0.001 \mathrm{~mm}$ [25]. Conventional UT sensors utilise a liquid couplant for energy transfer from transducer to part and challenges related to maintaining optimal stand-off, sufficient couplant and orientation to the surface limit their deployment in automated applications. Wheel or roller ultrasonic probe designs [26] feature an outer acoustically matched conformable tire that supports wave propagation into the part with reduced or no couplant requirements. This, along with their ability to roll along a surface, ensuring optimum sensor-surface perpendicularity, makes them ideal for automated applications [27], [28]. From an ultrasonic measurement perspective welding is considered as a harsh environment as the elevated temperatures could damage the sensitive sensors. Furthermore, the surface conditions and high amounts of Electromagnetic Interference (EMI) produced by the welding equipment lead to a reduction in overall signal power and an increase in system noise which can contribute to inaccurate measurements. Therefore, a robust UT measurement system deployed during welding should be able to tolerate the aforementioned challenges introduced by the process.

The requirement for internal measurement sensor deployment in autonomous applications has driven research and development in areas such as sensor design for remote deployment, robotic deployment of such NDE sensors and the associated control and signal processing necessary. This paper presents contributions to the field of advanced manufacturing, through real-time process control of automated fusion welding parameters based on simultaneous forward-looking ultrasonic thickness mapping. A proof-of-concept demonstrator is introduced and subsequent characterisation of system performance is evaluated.

\section{Experimental system}

\subsection{Hardware}

A purpose designed automated GTAW welding cell was designed and configured for flexible advanced sensor inspired welding research, as demonstrated in Figure 1. Although the GTAW process was implemented due to equipment availability, the measurement and control system could be easily deployed and replicated for other welding processes such as GMAW and SAW. While modern GMAW welding power sources have synergic and parametric control of arc power and wire speed based on material thickness, this is less common on GTAW power sources which typically accept control directly of desired arc current. In traditional GTAW the general convention on the current required to sufficiently fuse a joint increases non-linearly with increasing material thickness 
[29]. Additionally, the optimum arc current for a given joint is affected by many parameters such as material, weld type, joint configuration and torch design. Therefore, to sufficiently control arc current, a suitable real-time conversion strategy from measured material thickness was researched, developed and characterized.

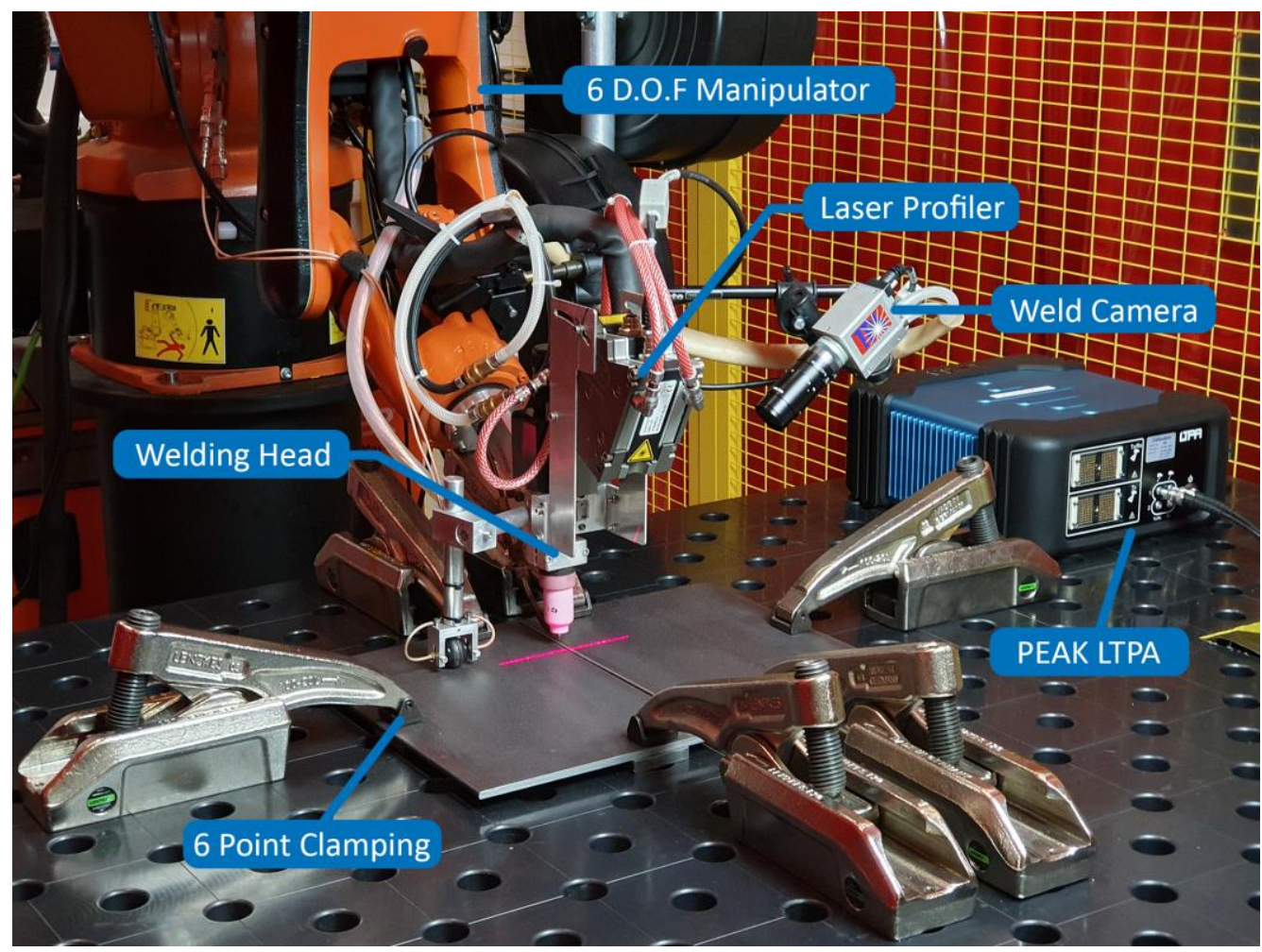

Fig. 1. Automated GTAW welding cell developed for feed forward control of welding parameters through in-process ultrasonic thickness measurement. A 6 DOF robotic manipulator is fitted with a welding head, laser profiler and weld camera. The PEAK LTPA ultrasonic driver and digitiser is located directly next to the location of welding.

A JÄCKLE/TPS ProTIG 350A AC/DC GTAW power source [30] with external control functionality provided the desired fusion arc energy. A JÄCKLE/TPS 4-roll wire feeder, with an optical encoder was powered and controlled via a dedicated system controller. Automation of the welding process was implemented using a KUKA KR5 - HW Arc 6 DOF [31] manipulator with a KRC2 controller, enabled with external control and Remote Sensor Interface (RSI) [32] for adaptive and real-time path control, and fitted with a custom TBI Industries welding torch. A High Dynamic Range Xiris weld camera focussed on the weld pool provided visual sensory information of the welding process. A MicroEpsilon scanCONTROL 2960/100 laser profiler [33] was used to locate the gap between the plates prior to welding them.

A National Instruments CRIO 9038 [34] embedded real-time controller was responsible for the developed control and data-logging tasks programmed in LabVIEW. All hardware within the automated welding cell, including the robotic manipulator, welding power source, wire feeder and ultrasonic digitizer, were controlled and interfaced in real-time through custom-code deployed on the cRIO. The software architecture contained multiple parallel processing loops, each ensuring optimum process flow and task allocation. The robotic welding torch position and orientation was calculated and updated at a 12 ms interpolation cycle through the KUKA RSI protocol, which also provided real-time positional feedback. All relevant welding power source parameters were interfaced through appropriate CRIO analogue and digital I/O modules for both control and logging 
purposes. The rotational speed of the wire-feeder motor was measured and controlled using Pulse Width Modulation through the NI 9505 DC servo drive, and was related appropriately to the desired control metric of linear wire feed speed.

\subsection{Ultrasonic Thickness Measurement}

Given the variation in plate thickness perpendicular to the weld does not directly affect process requirements for suitable weld joint penetration, a single-element automatically deployed ultrasonic wheel probe measurement was proposed to maximise process-speed and minimise surface interaction complexity. A custom $5 \mathrm{MHz}$ split-crystal dry coupled ultrasonic wheel probe, capable of measuring thicknesses between $2.5 \mathrm{~mm}$ and $100 \mathrm{~mm}$ [35], was fitted with high-temperature cables and was attached to the robot end-effector. A custom designed mount with 6 DOF adjustment allowed wheel probe to part optimum coupling, whilst offering flexible positioning around the welding torch. The designed robotic end-effector seen in Figure 2 was manufactured from Aluminium, which provided a good combination of light weight, durability and sufficient temperature resistance.
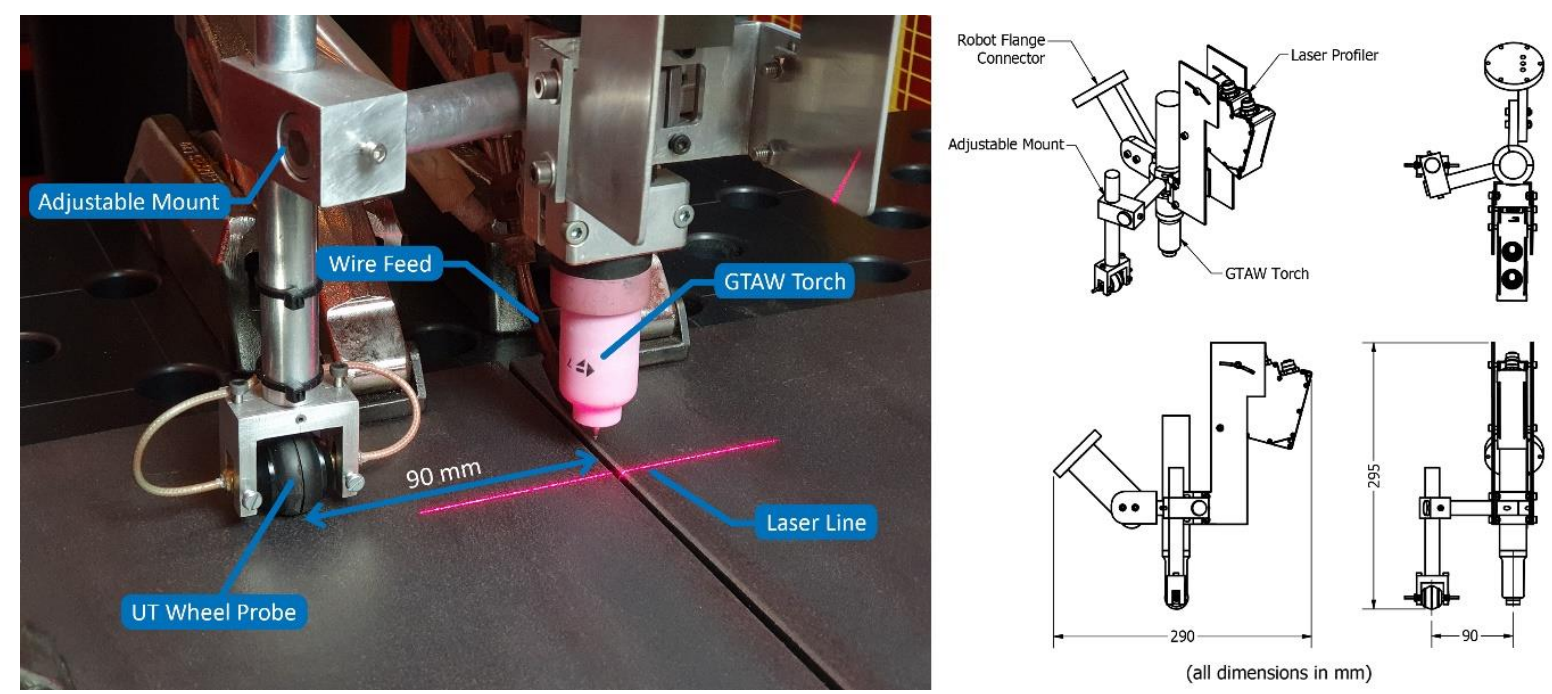

Fig. 2. Custom robotic end-effector for real-time feed-forward control of the GTAW process. An ultrasonic wheel probe, fitted with high temperature cables, is mounted $90 \mathrm{~mm}$ to the side of a GTAW welding torch using a custom adjustable mount. The gap between the two steel plates is detected through a projected laser line, in order to align the welding torch appropriately. All components of the end-effector were manufactured out of Aluminum to ensure light weight, durability and adequate temperature resistance.

The transducer required no external liquid coupling, as it consisted of two solid acoustically matched rubber tires rotating around a transducer housing to allow propagation of the transmitted compressional wave and receive signals into and from the base material (Figure 3. b)). The wheel probe had an approximate footprint size, and therefore lateral minimum spatial resolution, of $8 \mathrm{~mm}$. While the authors have researched and developed closed loop control of robotically delivered Phased Array wheel probes based of measurement of applied force [27], it was deemed that openloop control, based on robot end-effector distance to the base surface was sufficient for the probe utilised in this body of work. 


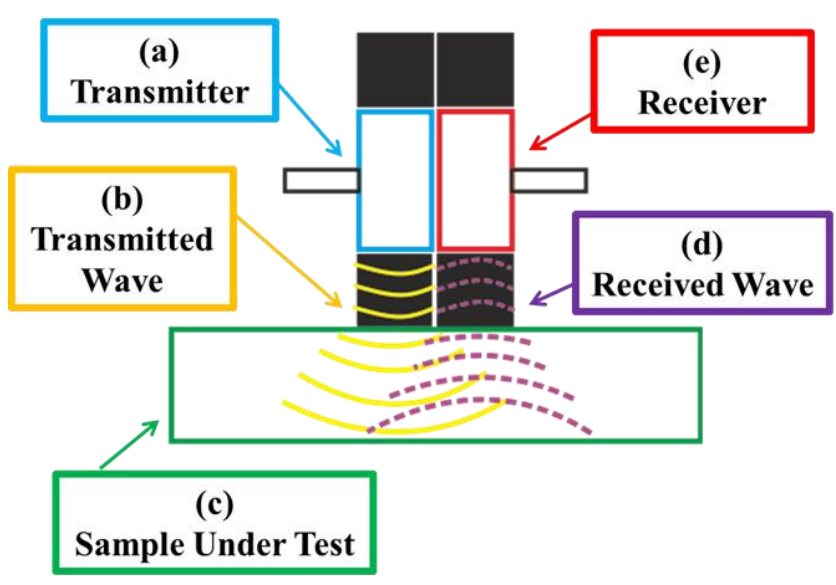

Fig. 3. Diagram of split piezoelectric crystal ultrasonic wheel probe with its key components: a) transmitting transducer; b) ultrasonic wave transmitted through the rubber tyre; c) sample under test; d) received echo of incident ultrasonic wave; e) receiving transducer.

As found in [28], due to the compressive nature of the wheel probe tyre and the difference in sound propagation velocity through the tyre and base material, accurate calculation of the thickness of the sample based on conventional time-of-flight information between the transmitted pulse and the first back-wall echo return was deemed unsuitable. Therefore, the corresponding thickness of the base material was determined using successive back-wall echoes, with three or more successive echoes utilised for averaging purposes. Furthermore, small variations in coupling between the tyre and surface could arise from reduced tyre to surface contact pressure, based on variations of robot end-effector to surface height variations, local surface anomalies and rubber tyre wear and tear. These variations of coupling level yield corresponding changes in the multiple back-wall echo amplitudes, with poorly coupled instances providing reduced amplitudes. The minimum peak detection amplitude of each back-wall echo must therefore take into consideration the coupling efficiency and the amplitude of waves physically transferred into the base material.

Delayed time gating of the received pulses excludes the initial trigger of the firing pulse and removes the valueless portion of the captured data, corresponding to the wave propagating through the tyre. Therefore, the largest peak in the acquired time window corresponds to the first back-wall echo, and each following peak corresponds to an internal reflection of the ultrasonic wave. Considering the attenuative nature of metallic materials, successive back-wall echoes are expected to have reduced amplitudes as depicted in Figure 4. The thickness of the tested material if, for example using three back-wall echoes, can be calculated through (1):

$$
m_{t}=\left(\frac{v_{c} \times\left(t_{3}-t_{1}\right)}{4}\right)
$$

Where $m_{t}=$ material thickness $(\mathrm{mm}), v_{c}=$ Speed of sound in material $(\mathrm{mm} / \mathrm{s}), t_{1}=$ time index of $1^{\text {st }}$ back-wall echo and $t_{3}=$ time index of $3^{\text {rd }}$ back-wall echo. The peak detection developed for this body of work, in terms sensitivity and robustness, adapts for variation in the amplitude of the actually transmitted wave and the amplitude of the first and a number of subsequent echoes. The algorithm and technique implemented in this paper required real-time calculation of material thickness for inthe-loop correction of welding parameters. 


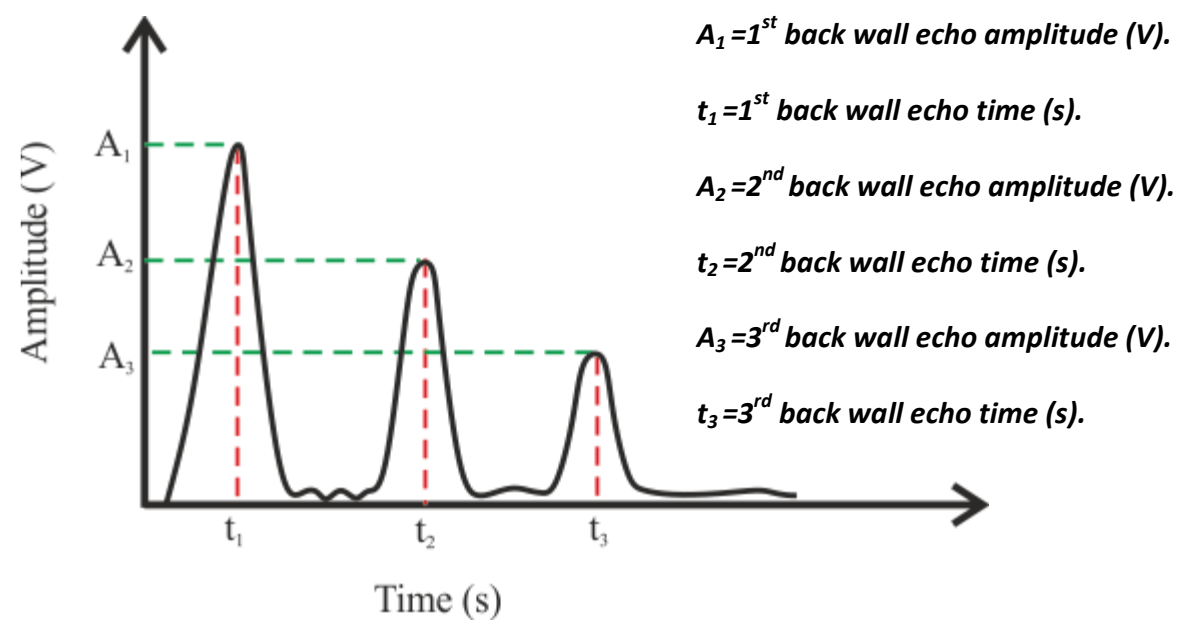

Fig. 4. Material thickness measurement through ultrasonic back-wall echoes. A number of successive wall reflections are utilised for averaging purposes. The thickness is measured based on the time-of-

flight between the first and last reflection and the speed of sound in the material under test.

The thermal propagation during GTAW welding across the range of applicable thicknesses was captured by measuring the surface temperature using a FLIR infrared thermal camera. As demonstrated in Figure 5, the surface temperature of the sample in its thinnest section ( $4 \mathrm{~mm}$ thick) was constant after a distance of $60 \mathrm{~mm}$ to the side of the welding torch. Therefore, the ultrasonic wheel probe was not subjected to any hazardous temperatures, capable of damaging the piezoelectric transducer (usually 60 ㅇ $\mathrm{C}$ or above), as it was positioned at a distance of $90 \mathrm{~mm}$ to the side of the welding torch. Moreover, as all ultrasonic measurements were taken at a location where the material was at uniform temperature, no measurement error arising from to changes in the speed of sound, due to temperature, were expected.

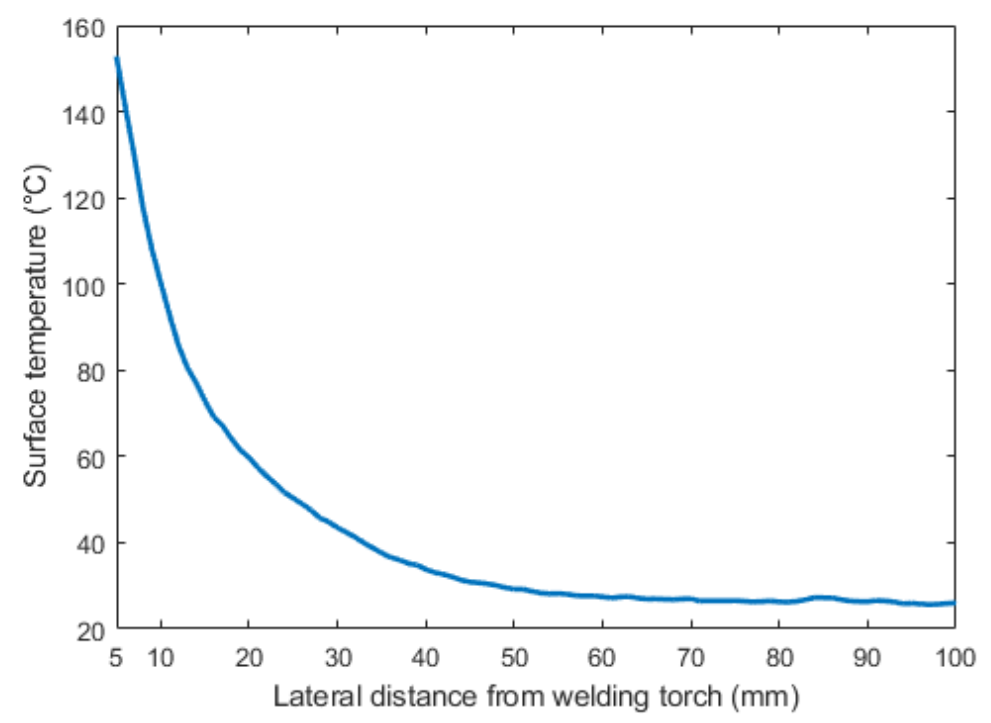

Fig. 5. Surface temperature of machined mild steel plate during GTAW fusion welding. Measurement was captured at a section, where the machined thickness of the plates was $4 \mathrm{~mm}$. All measurements were acquired using a FLIR infrared thermal imaging camera and show that no elevated temperatures are observed at lateral distances larger than $60 \mathrm{~mm}$ away from the welding torch. 


\subsection{Acquisition and Signal Processing}

The wheel probe transmitter and receiver were driven via a PEAK LTPA Ultrasonic Controller. The PEAK LTPA was configured to acquire 8-bit rectified A-scans at a Pulse Repetition Frequency (PRF) of $20 \mathrm{kHz} .128$ sample averaging and a $3 \mathrm{MHz}$ to $15 \mathrm{MHz}$ hardware bandpass filter were employed to reduce the electromagnetic noise emitted from the welding equipment and robotic manipulator. All received and recorded raw ultrasonic signals were digitized and transferred over Ethernet to the cRIO controller for further processing. Envelope detection was implemented using an Equiripple lowpass filter with a pass frequency of $3.9 \mathrm{MHz}$ and a stop frequency of $4 \mathrm{MHz}$. The peaks of the material back-wall reflections were detected using an adaptive detection threshold, in order to minimise the effect of any variations in coupling. The threshold utilized process feedback and was set as a proportion ( $25 \%$ ) of the largest detected peak amplitude, in order to capture a number of successive echoes. When considering the adaptive threshold, a minimum value of $10 \%$ screen height was utilised, to provide against falsely identifying system noise as back-wall echoes, in case no back-wall echoes were detected.

The developed code allowed for two or more peaks to be used for thickness calculation, as long as they satisfied a minimum $2^{\text {nd }}$ derivative value condition. This measure of peak "sharpness", ensured that lower amplitude peaks corrupted by noise did not affect the accuracy and repeatability of the final measurement. If fewer than two peaks satisfied this condition, meaning that material thickness could not be inferred from the ultrasonic signal, the last valid measurement was used for control purposes until this condition was once again satisfied. In the final step of the signal processing a median filter of 128 samples was applied to the measured thickness, removing any outlier measurements. The measured thickness was then used to calculate in real-time the welding current, welding torch travel speed and filler wire feed rate through pre-established relationships as shown in Figure 6. The welding current was externally set in the welding power source through a differential analogue voltage line. The welding travel speed and wire feed rate were internally set in the CRIO deployed LabVIEW software, updating the setpoints in the real-time motion control loop and PID motor control loop, respectively.

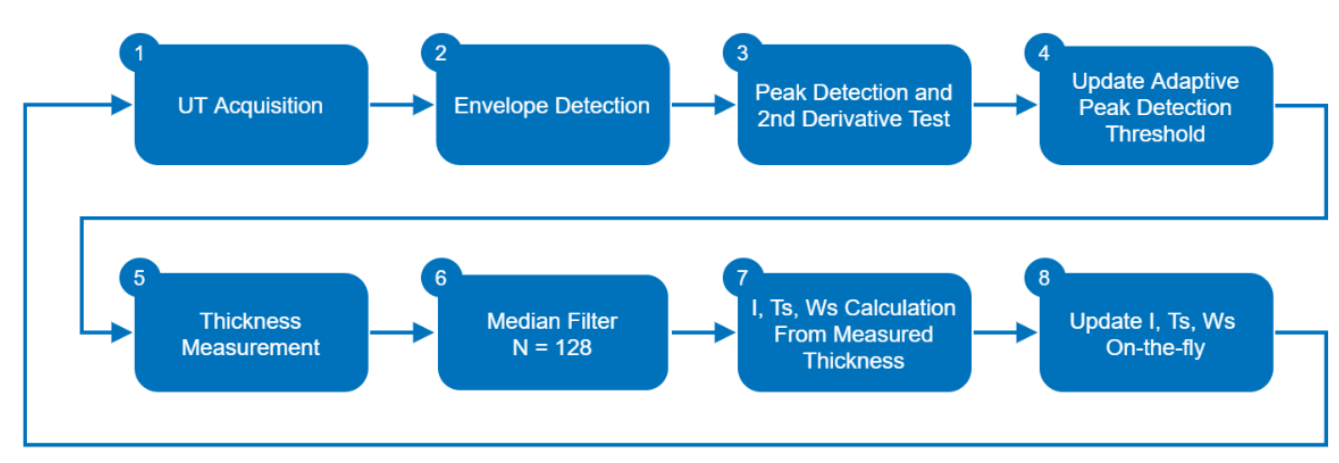

Fig. 6. Process flowchart for feed forward control of welding process parameters through on-line ultrasonic thickness measurement. Ultrasonic signals are acquired at a $20 \mathrm{kHz}$ PRF with 128 sample averaging, resulting in a $156.25 \mathrm{~Hz}$ overall update rate. An accurate material thickness measurement is extracted through envelope detection, adaptive peak detection and median filtering. The welding current, welding torch travel speed and filler wire feed rate are calculated and updated on-the-fly from the measured thickness and pre-established relationships.

As the acquisition and signal processing parameters were constant, the only variable that affected the spatial measurement resolution was the torch travel speed. Given the used PRF of $20 \mathrm{kHz}, 128$ 
sample averaging and the maximum welding torch speed used in the trials of $172 \mathrm{~mm} / \mathrm{min}$ (based on the maximum applicable weld plate thickness of $6.0 \mathrm{~mm}$ ), the surface length covered by a single measurement could be calculated by Equation (2):

$$
\text { spatial resolution }=\frac{T_{S^{*}} N_{\text {avg }} * N_{\text {med }}}{P R F * 60}
$$

Where $N_{\text {avg }}=$ number of acquisition averages and $N_{\text {med }}=$ median filter length. Therefore, the minimum spatial resolution for weld parameter control was $2.3 \mathrm{~mm}$ in the worst case. The response time of the welding equipment should also be considered and accounted for in very high-accuracy applications. The position of each thickness measurement was encoded through the robotic manipulator and was logged from the cRIO at $12 \mathrm{~ms}$ intervals. As the distance between the wheel probe contact point and the robotic welding torch in both $\mathrm{X}$ and $\mathrm{Y}$ axes was known and constant, each measurement was recorded relative to the correct location along the sample.

\subsection{Control Strategy And Calibration}

A control strategy was designed to demonstrate how the arc current, torch travel speed and wire feed rate can be updated on-line based on the measured sample thickness. Data from available reference thickness current ranges for manual GTAW butt-welding of mild steel [29] was used as initial starting points for developing the arc current-thickness parameter relationship (Figure 7, marked with ' $o$ ' and ' $x$ '). Due to material thickness having a direct nonlinear relationship to the arc current, a polynomial was selected to best fit all reference current range data points. Through inspection of the data in MATLAB it was established that a third order polynomial (Figure 7, dashed line) sufficiently captured the overall curve trends without overfitting the reference data points.

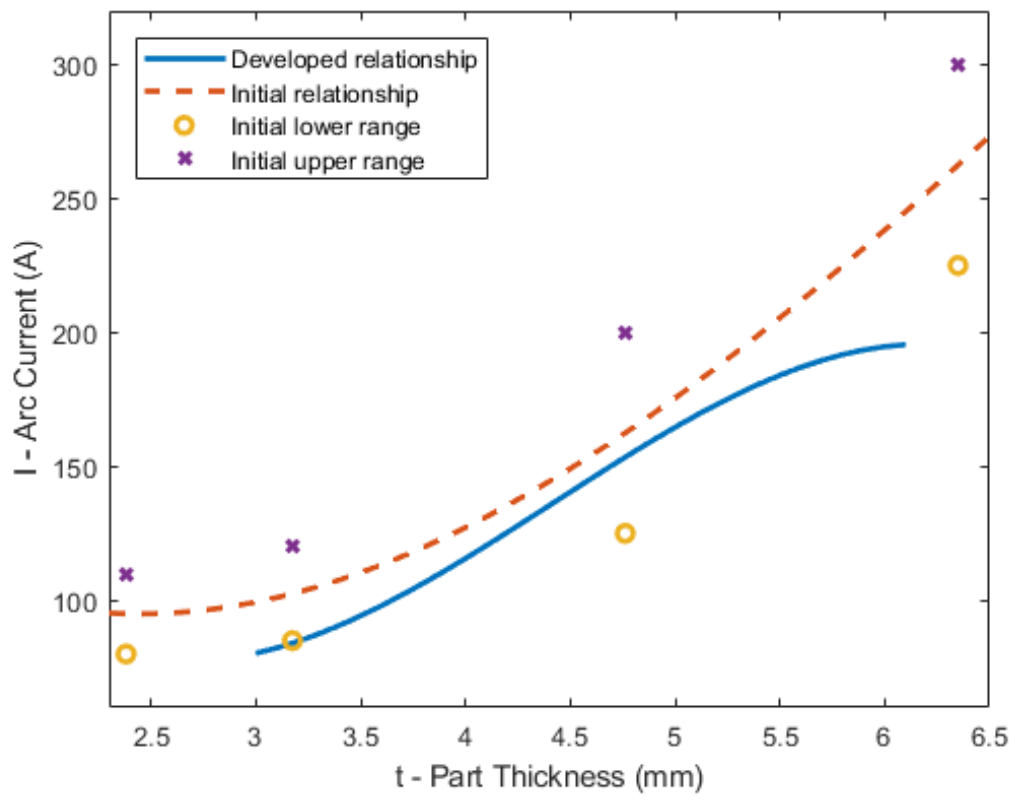

Fig. 7. Reference arc current values for manual GTAW butt-welding of mild steel [29] represented as a range with lower bound (marked with ' $o$ ') and upper bound (marked with ' $x$ '). A third order polynomial (dashed line) was fitted to the reference points and was used as a starting point for 
developing the final arc current-part thickness relationship for GTAW welding of mild steel (solid line).

The next step of the calibration process was to develop a control strategy for the torch travel speed. To reduce the number of variables and overall calibration complexity, it was decided to control the torch speed based on the arc current through a linear relationship, as the arc current was already directly controlled by the measured sample thickness. Therefore, in order to establish an initial arc current-torch speed relationship, two reference points were required. To obtain these, preliminary butt-weld trials with $6 \mathrm{~mm}$ and $3 \mathrm{~mm}$ thick plates were carried out. The arc current was set based on the initial thickness-arc current curve, and the torch travel speed and wire feed rate were manually adjusted until a satisfactory weld seam was achieved for both thicknesses. A linear function was then fitted between the two established torch travel speed points to generate the initial torch travel speed-thickness relationship. Finally, it was decided to maintain the wire feed rate proportional to the plate thickness and torch speed in order to provide a consistent and appropriate deposition rate.

To validate the concept, characterize the performance of the system, and optimise the initial relationships, a number of sample geometries were considered. Mild steel plates with a nominal thickness of $6 \mathrm{~mm}$ were selected, as they could be butt-welded in a single pass using the available GTAW setup. Computer Numerical Control (CNC) milling was used to machine different profiles out of the plates for calibration and for validation purposes. A calibration sample geometry was designed to feature a continuous slope ranging from the full thickness of the plate, to a $33 \%$ reduction of wall thickness, decreasing at a rate of $10 \mathrm{~mm}$ per $1 \mathrm{~m}$ as shown in the technical drawing at the bottom of Figure 8. The sloped calibration samples were welded in the direction of decreasing thickness so that the effects of the parameter relationships could be observed with respect to the reducing sample thickness. A number of calibration trials were carried out, each followed by a close inspection of the produced weld seam. Visual inspection was suitable for determining the overall weld quality, as this work was focused on demonstrating the adaptive deposition method, and not at obtaining any specific metallurgical qualities of the produced welds. Thus, the welding parameter relationships were iteratively updated based on a number of factors, e.g. amount of weld penetration, presence of undercut or burn-through, visible size of the HAZ and weld bead size. For example, if the deposited weld seam was consistent for thicknesses between $6 \mathrm{~mm}$ and $5 \mathrm{~mm}$ but then featured an excessive penetration for thicknesses below $5 \mathrm{~mm}$, the polynomial coefficients for obtaining the arc current would be manually optimised and adjusted in MATLAB, in order to provide a lower arc current for thicknesses below $5 \mathrm{~mm}$. This process was repeated until a consistent weld seam and penetration were successfully achieved along the full length of the weld, as shown in Figure 8. 


\section{Calibration sample}
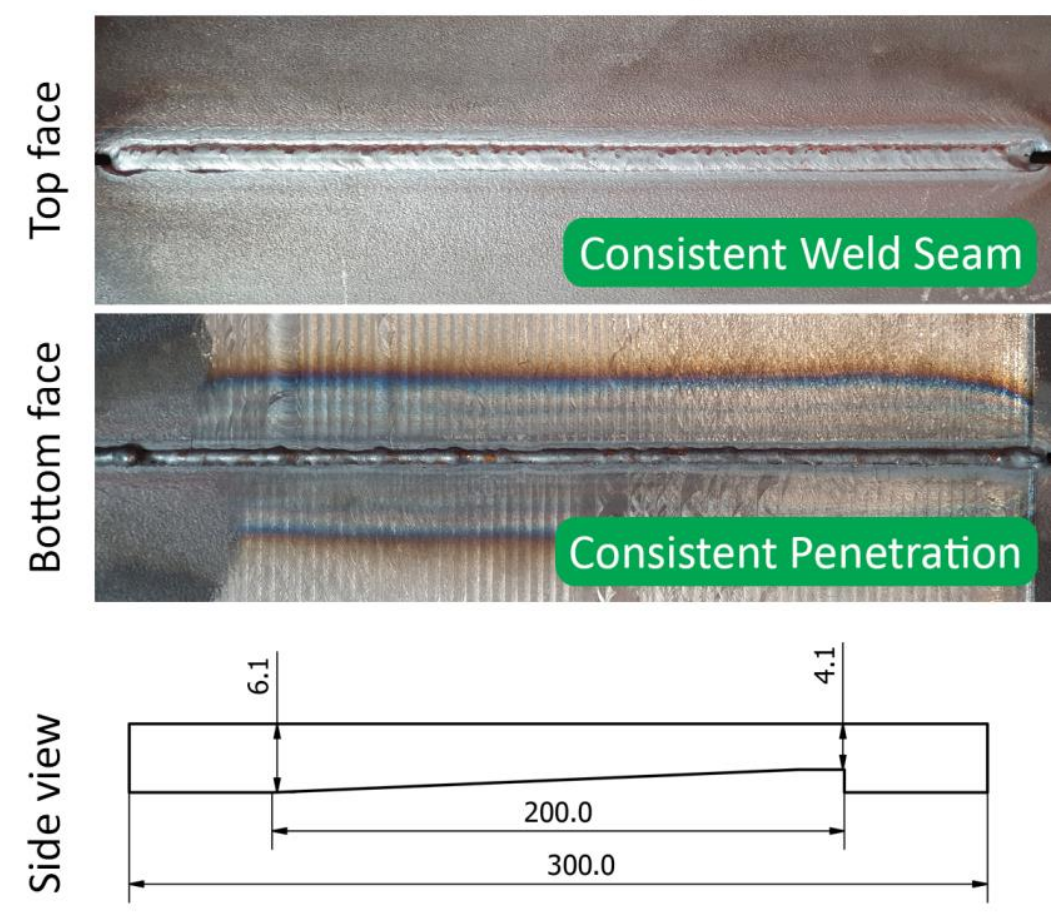

(all dimensions in $\mathrm{mm}$ )

Fig. 8. Calibration GTAW butt-weld of two mild steel plates with a machined slope representing wall thickness loss from $6.1 \mathrm{~mm}$ to $4.1 \mathrm{~mm}$ (measured using calibrated digital callipers). On-line ultrasonic thickness measurement and real-time control of welding arc, welding torch travel speed and filler wire feed rate were utilized to produce a consistent weld cap on the top face and consistent weld penetration on the bottom face of the plates.

The final forms of the developed welding parameter relationships after the calibration procedure are shown in Equations (3), (4) and (5), and the final arc current-thickness curve is plotted in Figure 7 (solid line).

$$
\begin{gathered}
I=-5.4 * t^{3}+71.58 * t^{2}-265.58 * t+379.4 \\
T_{S}=31.5+0.69 * I \\
W_{S}=3.6 * t * T_{S}
\end{gathered}
$$

Where $I=$ welding current $(\mathrm{A}), t=$ measured part thickness $(\mathrm{mm})$ and $W_{s}=$ filler wire feed rate $(\mathrm{mm} / \mathrm{min})$. Using the produced relationships, a preliminary estimate for arc current, torch travel speed and filler wire feed rate could be determined for GTAW butt-welding a given thickness of mild steel plates between $4.1 \mathrm{~mm}$ and $6.1 \mathrm{~mm}$. The thickness of each sample was measured both off-line, before any welding had commenced, and on-line, during the welding process, to determine the influence of the environmental noise introduced by the GTAW process. The RMS difference between the on-line and off-line measurements was $0.02 \mathrm{~mm}$ with a maximum discrepancy of $0.07 \mathrm{~mm}$ at any point. The above values take into account the measurement repeatability error introduced by various factors such as irregular tyre wear, robotic positioning error, EMI and background noise. 


\section{Validation And Results}

Further trials were carried out in order to test the developed parameters with a more realistic sample and to demonstrate its benefits over traditional automated welding systems. A second geometry weld profile was designed (sketched in Figure 9) featuring sections with various thicknesses between $6.1 \mathrm{~mm}$ and $4.1 \mathrm{~mm}$, where the wall thickness was increasing in some regions and decreasing in others at a rate of $30 \mathrm{~mm}$ per $1 \mathrm{~m}$. A control sample was manufactured with constant welding parameters set based on a single digital calliper measurement of the full plate thickness $(6.1 \mathrm{~mm}$ ) and Equations (3), (4) and (5), resulting in an arc energy of $1.2 \mathrm{~kJ} / \mathrm{mm}$. Figure $9 \mathrm{a}$ ) shows that the suboptimal arc energy used in the control sample has caused an undercut of the weld seam, and, in the thinner middle section, weld burn-through in the form of holes as seen from the top face. Moreover, paired with the constant wire feed rate, the bottom face of the produced weld was irregular with an excessive root penetration.

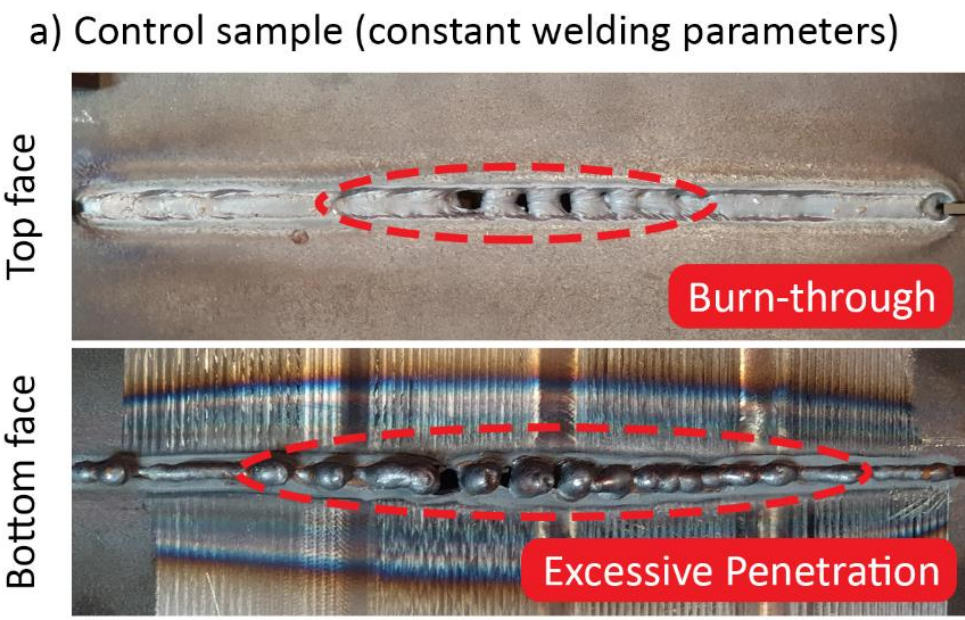

b) Validation sample (adaptive welding parameters)
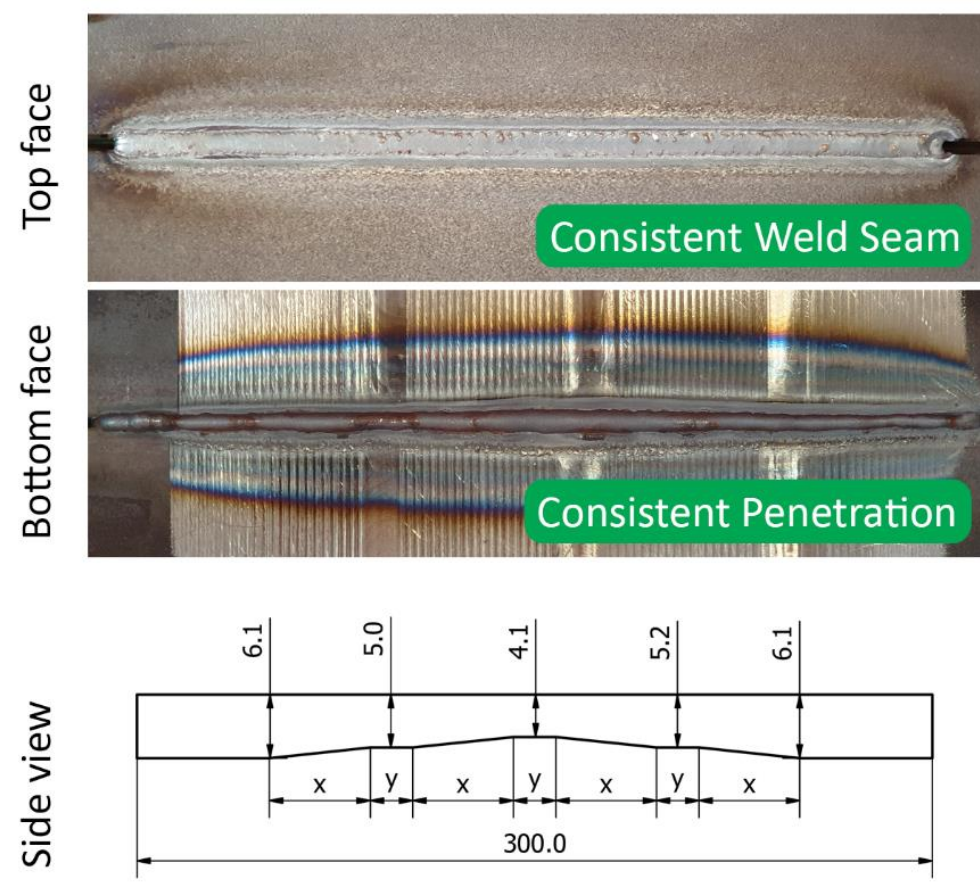

(all dimensions in $\mathrm{mm}$ ), $\mathrm{x}=38, \mathrm{y}=16$ 
Fig. 9. Control and validation samples machined down to the geometry sketched in the bottom; a)

Control sample manufactured with constant welding parameters assuming a constant plate thickness of $6.1 \mathrm{~mm}$, as obtained by a single point measurement with calibrated digital callipers. The suboptimal arc energy used in the control sample has caused an undercut and weld burn-through in the form of holes as seen from the top face and an irregular and excessive root penetration as seen from the bottom face; b) Validation sample manufactured with adaptive welding parameters based on ultrasonically measured sample thickness and the herein developed welding parameter relationships. The weld was of good visual appearance and had consistent weld bead and root penetration.

In contrast, the manufactured validation sample employing online thickness measurement in Figure $9 \mathrm{~b})$ exhibited both a consistent weld bead and root penetration. This was a result of the on-line welding parameter control, enabled by the ultrasonic wheel probe measurements. The significance of the obtained results becomes evident when looking at the measured thickness, arc current, torch travel speed and wire feed rate values plotted in Figure 10. For this sample, the arc energy was automatically adjusted on-the-fly between $0.8 \mathrm{~kJ} / \mathrm{mm}$ and $1.2 \mathrm{~kJ} / \mathrm{mm}$, equating to a total of $33.3 \%$ reduction between the thickest and thinnest section of the sample. The highest thickness measured by the ultrasonic wheel probe was $6.06 \mathrm{~mm}$, corresponding to an arc current of $203.6 \mathrm{~A}$, welding torch travel speed of $172 \mathrm{~mm} / \mathrm{min}$ and wire feed rate if $3832 \mathrm{~mm} / \mathrm{min}$. The lowest thickness measured was $4.12 \mathrm{~mm}$ at the thinnest middle section of the weld seam, with subsequently calculated arc current of $128.6 \mathrm{~A}$, torch travel speed of $120.3 \mathrm{~mm} / \mathrm{min}$ and wire feed rate of 1822 $\mathrm{mm} / \mathrm{min}$.
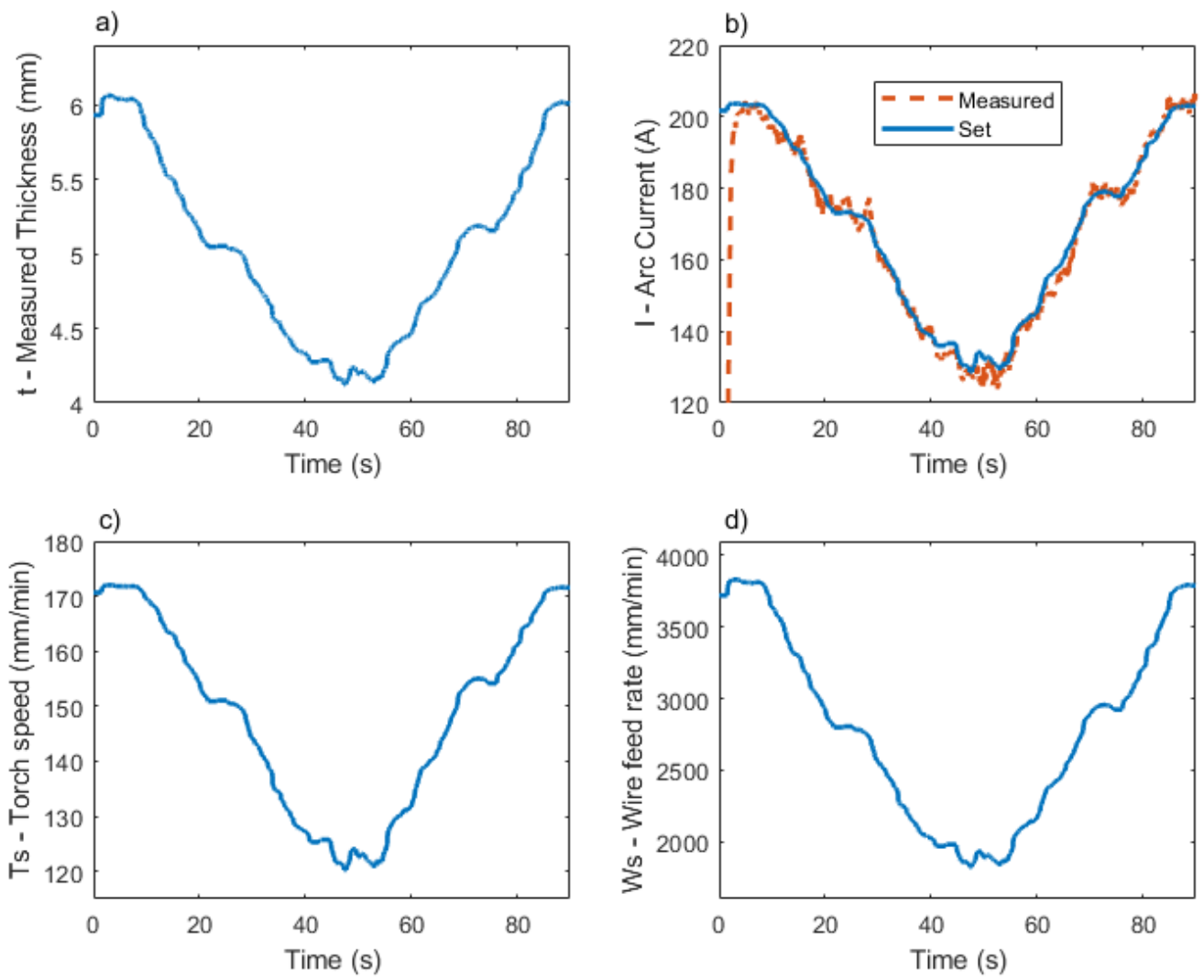
Fig. 10. On-the-fly welding parameter adjustment through ultrasonically measured thickness of sample with machined wall thickness loss: a) on-line measured sample thickness; b) set arc current

(solid) and measured arc current (dashed); c) welding torch travel speed; d) filler wire feed rate.

The results of the welding trials demonstrated that ultrasonic thickness measurement was fit for online welding parameter adjustment. The measurement accuracy given the harsh industrial environment, i.e. deployment during welding deposition, was sufficient and the control speed of the welding power source did not introduce any noticeable latency into the system. Furthermore, the developed system significantly outperformed current open-loop automated welding deposition, when considering parts with loss of wall thickness.

\section{Future Work}

Although all thickness measurements assumed a constant lateral thickness of the sample, the flexible mount for the ultrasonic wheel probe allowed for it to be positioned in front of the welding torch in order to measure the material thickness exactly at the weld interface. Moreover, if both plates are expected to change in thickness independently, of support varying thickness fillet welds, a second UT probe could be placed on the second plate and a measurement of the two readings could be used to calculate the required welding parameters and welding torch orientation. The real-time robot control through RSI makes it possible to adjust the welding torch orientation on the fly, in order to direct the welding arc as necessary. Therefore, the system can be employed with various welding processes and can be deployed in various situations and with different weld geometries, e.g. circumferential, fillet and lap among others. The proposed feed-forward system could also be deployed in conjunction with optical and other feed-back methods through sensor fusion, which would further increase the control over the final weld seam quality and metallurgical properties.

\section{Conclusion}

The work outlined in this paper has described the design and testing of a sensor-enabled automated GTAW welding system, employing an in-process ultrasonic thickness measurement and feed-forward welding parameter control of arc current, torch travel speed and wire feed. A number of significant contributions have been outlined in the paper:

Firstly, a novel sensing approach employing a robotically deployed ultrasonic wheel probe was described. The designed and manufacture custom 6 DOF mount attaching the wheel probe to the robotic end-effector allowed for the wheel probe to be positioned freely around the welding torch. Real-time signal processing was implemented to enable suppression of environmental noise emitted by the welding power source and manipulator robot, enabling on-line ultrasonic measurement of the welded sample.

Secondly, an ultrasonic thickness measurement algorithm was introduced that could accommodate variations in the distortions of the tyre of the wheel probe and in the sample surface to provide accurate estimations of thickness in real time.

Thirdly a parametric relationship between sample thickness and arc current, welding torch travel speed and filler wire feed rate for butt-welding S275 mild steel plates of thickness between $4.1 \mathrm{~mm}$ and $6.1 \mathrm{~mm}$ using the demonstrator system was established and verified.

It was demonstrated that closed-loop feed-forward control of arc current, torch travel speed and wire feed rate provided a consistent weld seam and uniform penetration on variable thickness steel samples. Furthermore, the developed approach can accommodate for changes in sample thickness, 
which under normal open-loop circumstances lead to sample burn-through and excessive penetration, leading to an improvement in terms of final product integrity, production rate and reduced re-work.

\section{Funding}

This work was funded by the Engineering and Physical Sciences Research Council (EPSRC), grant number 2096856.

\section{References}

[1] K. Weman and G. Lindén, Mig Welding Guide. Woodhead Publishing, 2006.

[2] D. Manufacturing Institute, "The skills gap in U.S. manufacturing 2015 and beyond." 2015.

[3] M. Hardie and A. Banks, "The Changing Shape of UK Manufacturing." The Office for National Statistics, Oct. 22, 2014.

[4] Airbus, "Flying by numbers: Global Market Forecast for 2015-2034." 2015.

[5] McKinsey \& Company, "The road to 2020 and beyond: What's driving the global automotive industry." Sep. 2013.

[6] IHS Global, "Impact of the Manufacturing Renaissance from Energy Intensive Sectors." 2013.

[7] Germany Trade \& Invest, "GTAI - Industrie 4.0 - What is it?," Germany Trade \& Invest. https://www.gtai.de/GTAl/Navigation/EN/Invest/Industries/Industrie-4-0/Industrie-40/industrie-4-0-what-is-it.html\#1798424 (accessed Sep. 02, 2019).

[8] K. Weman, "15 - Mechanisation and robot welding," in Welding Processes Handbook (Second edition), Woodhead Publishing, 2012, pp. 157-166.

[9] A. E. Öberg and E. Åstrand, "Variation in welding procedure specification approach and its effect on productivity," Procedia Manufacturing, vol. 25, pp. 412-417, Jan. 2018, doi: 10.1016/j.promfg.2018.06.111.

[10] T. Font comas, C. Diao, J. Ding, S. Williams, and Y. Zhao, "A Passive Imaging System for Geometry Measurement for the Plasma Arc Welding Process," IEEE Transactions on Industrial Electronics, vol. 64, no. 9, pp. 7201-7209, Sep. 2017, doi: 10.1109/TIE.2017.2686349.

[11] P. Ghanty et al., "Artificial neural network approach for estimating weld bead width and depth of penetration from infrared thermal image of weld pool," Science and Technology of Welding and Joining, vol. 13, no. 4, pp. 395-401, May 2008, doi: 10.1179/174329308X300118.

[12] X. Li, X. Li, S. S. Ge, M. O. Khyam, and C. Luo, "Automatic Welding Seam Tracking and Identification," IEEE Transactions on Industrial Electronics, vol. 64, no. 9, pp. 7261-7271, Sep. 2017, doi: 10.1109/TIE.2017.2694399.

[13] Yuan Li, You Fu Li, Qing Lin Wang, De Xu, and Min Tan, "Measurement and Defect Detection of the Weld Bead Based on Online Vision Inspection," IEEE Transactions on Instrumentation and Measurement, vol. 59, no. 7, pp. 1841-1849, Jul. 2010, doi: 10.1109/TIM.2009.2028222.

[14] Y. M. Zhang, R. Kovacevic, and L. Li, "Adaptive control of full penetration gas tungsten arc welding," IEEE Transactions on Control Systems Technology, vol. 4, no. 4, pp. 394-403, Jul. 1996, doi: $10.1109 / 87.508887$.

[15] Z. Wang, "An Imaging and Measurement System for Robust Reconstruction of Weld Pool During Arc Welding," IEEE Transactions on Industrial Electronics, vol. 62, no. 8, pp. 5109-5118, Aug. 2015, doi: 10.1109/TIE.2015.2405494.

[16] Y. Liu, W. J. Zhang, and Y. Zhang, "Estimation of Weld Joint Penetration under Varying GTA Pools," 2013. /paper/Estimation-of-Weld-Joint-Penetration-under-Varying-LiuZhang/a147600842ec3d598af4182201503642251f26ec (accessed Jan. 17, 2021).

[17] Y. Liu and Y. Zhang, "Control of 3D weld pool surface," Control Engineering Practice, vol. 21, no. 11, pp. 1469-1480, Nov. 2013, doi: 10.1016/j.conengprac.2013.06.019. 
[18] R. K. W. Vithanage et al., "A phased array ultrasound roller probe for automated inprocess/interpass inspection of multipass welds," IEEE Transactions on Industrial Electronics, pp. 1-1, 2020, doi: 10.1109/TIE.2020.3042112.

[19] L. C. Lynnworth, Ultrasonic Measurements for Process Control: Theory, Techniques, Applications. Academic Press, 2013.

[20] T. Pfeifer and M. Benz, "Ultrasonic on-machine measurement for internal topographies," International Journal of Production Research, vol. 40, no. 15, pp. 3821-3834, Jan. 2002, doi: 10.1080/00207540210133525.

[21] P. Gao, C. Wang, Y. Li, and Z. Cong, "Electromagnetic and eddy current NDT in weld inspection: A review," Insight - Non-Destructive Testing and Condition Monitoring, vol. 57, Jun. 2015, doi: 10.1784/insi.2015.57.6.337.

[22] B. P. C. Rao, B. Raj, T. Jayakumar, and P. Kalyanasundaram, "An artificial neural network for eddy current testing of austenitic stainless steel welds," NDT \& E International, vol. 35, no. 6, pp. 393398, Sep. 2002, doi: 10.1016/S0963-8695(02)00007-5.

[23] T. Kiwa, H. Tahara, E. Miyake, H. Yamada, and K. Tsukada, "Non-Contact Thickness Gauge for Conductive Materials Using HTS SQUID System," IEEE Transactions on Applied Superconductivity, vol. 19, no. 3, pp. 801-803, Jun. 2009, doi: 10.1109/TASC.2009.2019196.

[24] Y. Yu, Y. Zou, M. A. Hosani, and G. Tian, "Conductivity Invariance Phenomenon of Eddy Current NDT: Investigation, Verification, and Application," IEEE Transactions on Magnetics, vol. 53, no. 1, pp. 1-7, Jan. 2017, doi: 10.1109/TMAG.2016.2616328.

[25] OLYMPUS, "45MG Ultrasonic Thickness Gage." https://www.olympusims.com/en/45mg/\#!cms[focus]=cmsContent11047 (accessed Feb. 11, 2020).

[26] B. Drinkwater and P. Cawley, "An Ultrasonic Wheel Probe Alternative to Liquid Coupling," in Review of Progress in Quantitative Nondestructive Evaluation: Volume 14, D. O. Thompson and D. E. Chimenti, Eds. Boston, MA: Springer US, 1995, pp. 983-989.

[27] C. Mineo et al., "Fast ultrasonic phased array inspection of complex geometries delivered through robotic manipulators and high speed data acquisition instrumentation," in 2016 IEEE International Ultrasonics Symposium (IUS), Sep. 2016, pp. 1-4, doi: 10.1109/ULTSYM.2016.7728746.

[28] C. N. Macleod, G. Dobie, S. G. Pierce, R. Summan, and M. Morozov, "Machining-Based Coverage Path Planning for Automated Structural Inspection," IEEE Transactions on Automation Science and Engineering, vol. 15, no. 1, pp. 202-213, Jan. 2018, doi: 10.1109/TASE.2016.2601880.

[29] MillerWelds, "TIG Weld Setting Calculator." https://www.millerwelds.com/resources/weldsetting-calculators/tig-welding-calculator (accessed Mar. 05, 2020).

[30] Jäckle Schweiß- und Schneidtechnik GmbH, "Jackle ProTIG 350AC / DC Operating Manual." [Online]. Available: https://www.jaeckleess.com/out/media/BeOp-ProTIG-350-AC-DC-V10(3).pdf.

[31] KUKA Robot Group, "KR 5 arc HW, KR 5 arc HW-2 Specification." Mar. 23, 2016, Accessed: Sep. 05, 2019. [Online]. Available: https://www.kuka.com/-/media/kukadownloads/imported/48ec812b1b2947898ac2598aff70abc0/spez_kr_5_arc_hw_en.pdf?rev=2 c03c098785645f4a9fe488936fa5f10? modified $=-1090730564$.

[32] "KUKA.RobotSensorInterface," Apr. 2014. http://www.kuka-robotics.com.

[33] Micro-Epsilon, "Compact laser scanner for high precision." https://www.microepsilon.co.uk/2D_3D/laser-scanner/scanCONTROL-2900/ (accessed Mar. 05, 2020).

[34] National Instruments, "cRIO-9038." http://www.ni.com/en-gb/support/model.crio-9038.html (accessed Sep. 05, 2019).

[35] Eddyfi, "R-scan Manual Ultrasonic System." https://eddyfi.com/en/product/rscan-manualultrasonic-system (accessed Mar. 05, 2020). 